\title{
Review Article Noncoding RNA and Cardiomyocyte Proliferation
}

\author{
Shuang Qu, Chunyu Zeng, and Wei Eric Wang \\ Department of Cardiology, Chongqing Institute of Cardiology \& Chongqing Cardiovascular Clinical Research Center, \\ Daping Hospital, Third Military Medical University, Chongqing 400042, China \\ Correspondence should be addressed to Chunyu Zeng; chunyuzeng01@163.com and Wei Eric Wang; weiericwang@163.com
}

Received 30 June 2017; Accepted 20 August 2017; Published 31 October 2017

Academic Editor: Yaoliang Tang

Copyright (c) 2017 Shuang Qu et al. This is an open access article distributed under the Creative Commons Attribution License, which permits unrestricted use, distribution, and reproduction in any medium, provided the original work is properly cited.

It is acknowledged that postnatal mammalian cardiomyocytes (CMs) turn over with a very limited efficacy in both physiological and pathological conditions. Recent studies showed that those newly formed CMs are derived from preexisting CMs. Thus, stimulating CM proliferation becomes a promising strategy for inducing cardiac regeneration. Noncoding RNAs were found differently expressed in CMs with different proliferation potential. Moreover, manipulation of noncoding RNAs, in particular microRNAs, was proved to promote or suppress CM proliferation, indicating that noncoding RNAs are involved in the underlying mechanism of CM proliferation. This review mainly summarizes the roles of noncoding RNAs, as a class of influential factors, in the regulation of CM proliferation.

\section{Introduction}

Cardiovascular diseases are the leading causes of morbidity and mortality all over the world. Particularly, myocardial infarction (MI) and heart failure following myocardial ischemia can lead to a large number of CM death [1]. In the past, the adult mammalian CMs are regarded as terminally differentiated cells without the ability to proliferate. Fetal CMs proliferate during development but lose this ability quickly after birth, and myocardium goes through a hyperplastic to hypertrophic transition. After this transition, the predominant form of growth is an increase in cell size and myofibril density rather than the number of CMs [2].

It is now recognized that a low level of postnatal CM proliferation was demonstrated in both normal and injured hearts. Taking advantage of integration of ${ }^{14} \mathrm{C}$ into DNA to establish the age of CMs in human, a seminal study carried by Bergmann and his colleagues indicated that about $0.5-1 \%$ of CMs renews every year, so nearly $50 \%$ of CMs is replenished over a life span [3]. Recently, a combination of genetic fate mapping with stable isotope labeling and multi-isotope imaging mass spectrometry shows the renewal of CMs is predominantly from the division of preexisting CMs, rather than the differentiation from the stem cells or progenitors [4]. Our previous study showed that mature adult CMs can reenter the cell cycle and form new CMs through a threestep process, dedifferentiation, proliferation, and redifferentiation [5]. However, the proliferation is not enough to replenish the lost CMs and repair the injured myocardium, and the underlying mechanism regulating $\mathrm{CM}$ proliferation is still unclear. To decipher the molecular mechanism controlling CM proliferation is of great importance for stimulating the endogenous cardiac regeneration, which might be a new therapeutic approach to those patients suffering from heart diseases.

Noncoding RNAs are those RNAs which cannot code proteins, such as microRNAs (miRNAs), long noncoding RNAs (lncRNAs), and circular RNAs, and were found to play important roles in the regulation of multiple cellular activities including proliferation [6]. This review mainly summarizes the roles of noncoding RNAs in the regulation of mammalian CM proliferation.

\section{The Role of miRNAs in CM Proliferation}

MicroRNA is a small noncoding RNA molecule containing $20 \sim 24$ nucleotides. Each miRNA can have multiple target genes, and it can have various spatial and temporal expression patterns which express differently in diverse tissues and developmental stages [7]. An miRNA array showed that, 
among the over 1000 miRNAs analyzed, 204 miRNAs increased and 311 miRNAs decreased during neonatal rat CM proliferation [8]. miRNAs were demonstrated to influence CM proliferation in neonatal and adult stages, which were summarized in Table 1.

\section{3. miRNAs Regulate Neonatal CM Proliferation}

The proliferation capacity of mammalian CM is robust in fetal period and is switched off early after birth. In mouse, the 1-day-old neonatal hearts can regenerate after partial surgical resection, but this capacity is lost by 7 days of age [9].

MiR-499 is a miRNA which is abundantly found in CMs and almost does not express in human cardiac stem cells or human embryonic stem cells [10]. By transfecting with premiR-499, EdU incorporation indicated CM proliferation was increased by $50 \%$ [11]. MiR-499 displayed a highlighted ability to promote neonatal CM proliferation via its function on Sox6 and cyclin D1 [12]. Sox6 played a role in cell viability, inhibited cell proliferation, and promoted cell apoptosis [13]. MiR-410 and miR-495 both belong to Gtl2-Dio3 miRNAs and were reported to promote CM proliferation. Overexpressing miR-410 and miR-495 in NRVMs induced about a 2.5-fold increase of proliferation analyzed with EdU incorporation assay. Meanwhile, Ki-67 immunostaining showed a threefold increase of proliferation [14]. The target gene of miR-410 and miR-495 is Cited2, a coactivator required for proper cardiac development. Cited 2 knockdown reduced the expression of cell cycle inhibitor Cdkn1c/p57/ Kip2 in neonatal CMs [14]. In the ischemic injury model, miR-222-overexpressing mice showed a twofold phosphohistone 3 (PH3) CMs compared with controls. Inhibition of miR-222 in vivo blocked CM proliferation in response to exercise. Cell cycle inhibitor P27, HIPK-1, and HIPK-2 as well as HMBOX1 were found to be involved in miR-222induced CM proliferation [15].

MiR-133a knockdown mice hearts showed excessive CM proliferation, while miR-133a overexpression transgenic mice showed a diminished CM proliferation, indicating that miR-133 could be an inhibitor of CM proliferation [16, 17]. Similarly, miR-29a also suppressed CM proliferation, while inhibiting miR-29a promoted CM division [18]. Inhibiting miR-29a in neonatal CMs promoted CM proliferation by threefold analyzed by Ki-67 and $\mathrm{PH} 3$ staining and decreased the number of CMs in G0/G1 phases, while increased proportion of CMs in S and G2/M phases, indicating that inhibition of miR-29a facilitates the transition of G1/S and G2/M in CMs [19].

\section{4. miRNAs Regulate Adult CM Proliferation}

Hsa-miR-590-3p and hsa-miR-199a-3p are found to induce the proliferation of not only neonatal CMs but adult CMs. By injecting synthetic miRNAs directly into the heart of neonatal mice, EdU incorporation analysis revealed a marked increase of CM proliferation. Injection of AAV9 vector-expressing hsa-miR-590 or hsa-miR-199a precursor miRNAs increased CM proliferation in both neonatal and adult mice [8]. These two miRNAs can also stimulate CM proliferation in post-MI heart, which contributes to the preserved cardiac function [8].

Overexpressing miR-204 improved CM proliferation in neonatal and adult mice CMs in vitro. Knockdown of its target gene Jarid2 had a similar effect as miR-204 overexpression. Transgenic mice with cardiac-specific overexpression of miR-204 showed an increase of CM proliferation throughout the embryonic and adult stages, which was associated with upregulated cell cycle regulators Cyclin A, Cyclin B, Cyclin D2, Cyclin E, CDC2, and PCNA [20].

MiR-17-92, an oncogenic miRNA cluster, proved to be essential for CM proliferation and participated in the regulation of CM proliferation in embryonic, postnatal, and adult hearts. CM proliferation decreased by about $50 \%$ in postnatal hearts of miR-17-92 cKO mice, while it was significantly increased in cardiac-specific miR-17-92 overexpressed transgenic mice analyzed with $\mathrm{PH} 3$ and Aurora B immunostaining [21]. Overexpression of MiR-17-3p, a member of miR-17-92 cluster, has been shown to promote CM proliferation in neonatal CMs. Furthermore, inhibition of miR-17-3p attenuated exercise-induced cardiac growth and CM proliferation in adult mouse heart [22].

Loss of miR302-367 led to decreased CM proliferation during development, while increased miR302-367 expression led to a profound increase in CM proliferation. Reexpression of miR302-367 by using miRNA mimic-based treatment promoted adult CM proliferation and reduced scar formation in the post-MI heart. The CM proliferation was evaluated with Ki-67, PH3, and Aurora B kinase staining, as well as CM number counting [23]. Besides, miR-302-367 can not only have an effect on cell cycle activity but also the nucleation of CMs evidenced by an increase of the proportion of mononucleated/binucleated CMs versus multinucleated CMs in miR302-367 gain of function mice. With the method of high-throughput sequencing of RNA isolated by cross-linking immunoprecipitation (HITS-CLIP), the miR302-307 target genes Mst1, Lats2, and Mob1b are found to be components of Hippo signaling pathway, indicating the effect of miR302-367 on CM proliferation might be through repression of the Hippo signal transduction pathway [24].

MiR-34a expressed at a low level in fetal and early postnatal hearts; it soon expressed relatively higher after the first week after birth and sustained during adulthood [25]. Cardiac injury further upregulated the expression of miR34a [26]. In the early postnatal mice, overexpression of miR-34a can decrease the CM proliferation. In contrary, antagonism of miR-34a promoted the CM proliferation through targeting on Bcl2, Cyclin D1, and Sirt 1 in the adult mice with MI injury [25]. Overexpression of miR-195, a member of the miR-15 family, during development caused premature CM cell cycle arrest, leading to congenital heart hypoplasia [27]. MiR-195 was found to be upregulated by sixfold at postnatal day 10 compared to postnatal day 1 [28]; miR-195 overexpression prevented cardiac regeneration of postnatal day 1 hearts suffering from MI injury [27]. Overexpressing miR-195 increased the proportion of NRVMs in G2/M phase [28]. In adult MI model, inhibition of miR-15 family by using administrating-locked nucleic acid- (LNA-) modified anti-miRNAs resulted in an increase 


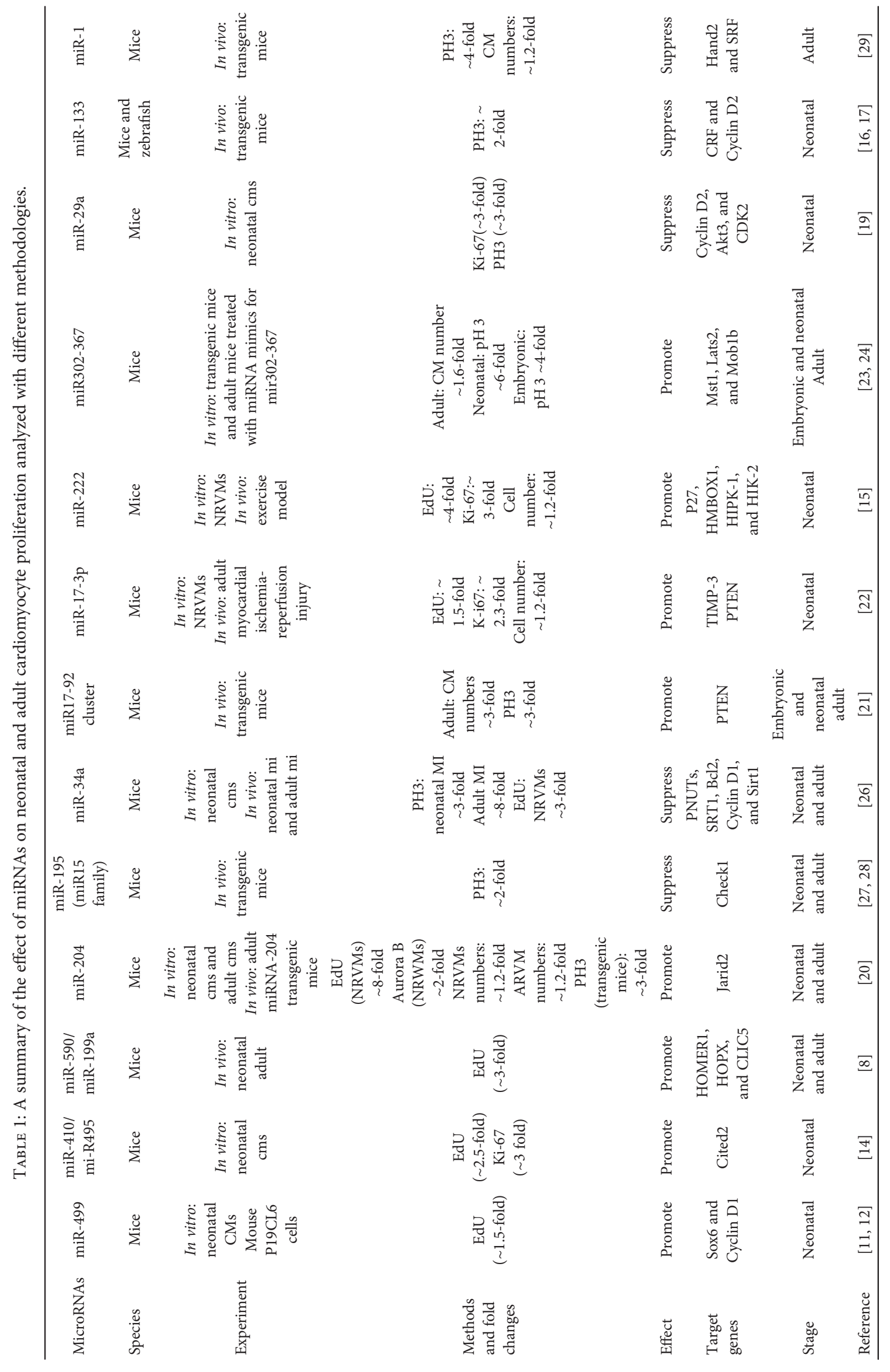




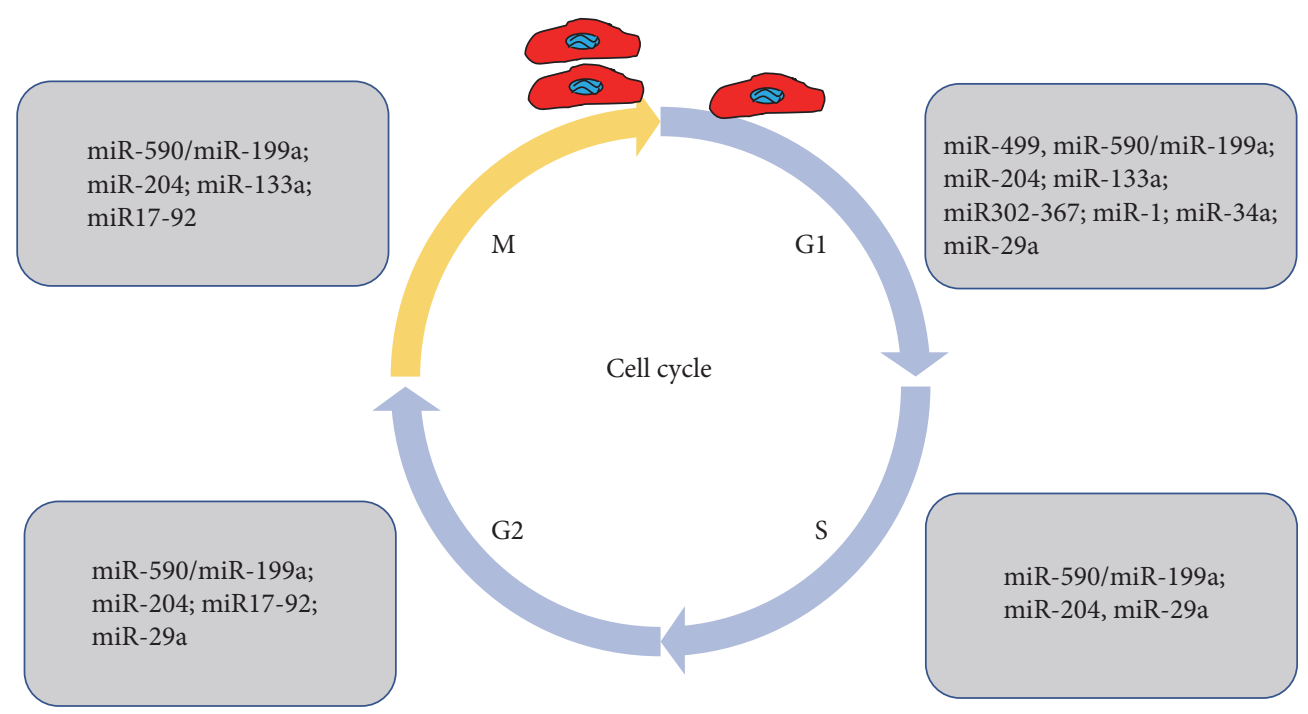

FIGURE 1: A summary of miRNAs in regulating cardiomyocyte proliferation at different phases of cell cycle.

in $\mathrm{CM}$ proliferation, with a fivefold increase of $\mathrm{PH} 3$-positive CMs [27]. Consistently, a chemically modified RNA oligonucleotide blocking the seed sequence of the miR-15 family members promoted adult CM proliferation and preserved cardiac function after injury [18]. In mice with miR-1-2 deletion, CM proliferation was increased, supported by $20 \%$ increased $\mathrm{CM}$ numbers and threefold more $\mathrm{PH} 3$ positive CMs [29].

\section{Cell Cycle and Methodology for Evaluating CM Proliferation}

Cell cycle can be divided into four phases: G1 phase, S phase (synthesis), G2 phase (collectively known as interphase), and mitotic (M) phase. Mitosis (division of the nucleus) and cytokinesis (division of cytoplasm, organelles, and cell membrane) together define the M phase of CM cell cycle [30]. The process of mitosis is divided into five stages: prophase, prometaphase, metaphase, anaphase, and telophase. It leads to multinucleated CMs if there is karyokinesis without cytokinesis during $\mathrm{M}$-stage, and it leads to polyploid if there is DNA replication without karyokinesis and cytokinesis [31]. When considering the intrinsic proliferative capacity of adult mammalian CMs, it is important to reiterate that DNA synthesis does not necessarily result in genome duplication, that genome duplication does not necessarily result in mitosis, and that mitosis does not necessarily result in cytokinesis [32]. miRNAs regulate CM proliferation at different phases of cell cycle, which were summarized in Figure 1.

In most studies, the methods used for quantifying $\mathrm{CM}$ proliferation were based on immunostaining for DNA synthesis and cell cycle activation (BrdU/EdU incorporation, Ki-67, PH3, Aurora B, and alinin). BrdU/EdU can be incorporated into newly synthesized DNA at S phase of cell cycle. $\mathrm{Ki}-67$ is detected in all active stages of cell cycle, including G1, S, G2, and $M$ phases and is not active in the resting G0-phase cells and terminally differentiated cells [33]. K-i67 could not clearly distinguish multinucleated cells and polyploid with proliferating cells. Aurora B kinase is a protein that functions in the attachment of the mitotic spindle to the centromere, which is expressed during metaphase, anaphase and, cytokinesis in CMs [34]. In metaphase, Aurora B is associated to the chromosomes, whereas in anaphase and telophase it is localized to the midzone and midbody, respectively [34]. PH3 expresses during mitosis since chromosome condensation at mitosis is accompanied not only with phosphorylation of histone H3. Actually, all these markers could not identify a CM completing the whole cell cycle process and giving rise to two/multiple daughter cells. CMs especially adult ones are able to reenter into cell cycle but difficult to pass cytokinesis phase. However, none of these markers directly examine CM proliferation with cytokinesis. On the other hand, these methods could be complicated by DNA repair, polyploidy, and multinucleation in CMs. One must keep the interpretive restrictions in mind when comparing the results from different laboratories particularly when different assays are utilized.

Therefore, rigorous confirmation with nonambiguous molecular genetic markers or method should be requisite for any studies assessing de novo cardiomyogenesis. Timelapse imaging observation combined with nuclei staining is a direct way to assess the CMs' proliferation and visualize the mitosis and cytokinesis processes [5]. This method could be an appropriate in vitro experiment, but new techniques are needed to quantify the CM proliferation in vivo.

\section{Discussion}

Understanding the underlying mechanism regulating CM proliferation could be of great clinical significance for treating MI, heart failure, and other cardiac diseases in which reduced CM numbers are the principal reason for deranged cardiac function. Noncoding RNAs especially miRNAs were demonstrated to play essential roles in CM proliferation in fetal and neonatal as well as adult stages. Manipulating the key miRNAs could be a promising strategy for stimulating 
cardiac regeneration in injured hearts. Other noncoding RNAs such as lncRNAs (with more than 200 nucleotides in length) may also play a role in CM proliferation. LncRNA expressions significantly changed in cardiac hyperplastic to hypertrophic growth transition [35]. Manipulation of lncRNA-Gas5 and Sghrt in adult heart reduced the expression of cell cycle regulating genes including Ccngl and Ccnd2 in CMs [36]. The effect of these noncoding RNAs including lncRNAs and circular RNAs on CM proliferation requires further study.

\section{Conflicts of Interest}

The authors declare that they have no conflicts of interest.

\section{Acknowledgments}

This study was supported by research grants from the National Natural Science Foundation of China (31730043 and 81670277).

\section{References}

[1] N. J. Pagidipati and T. A. Gaziano, "Estimating deaths from cardiovascular disease: a review of global methodologies of mortality measurement," Circulation, vol. 127, no. 6, pp. 749-756, 2013.

[2] E. R. Porrello, A. I. Mahmoud, E. Simpson et al., "Transient regenerative potential of the neonatal mouse heart," Science, vol. 331, no. 6020, pp. 1078-1080, 2011.

[3] O. Bergmann, R. D. Bhardwaj, S. Bernard et al., "Evidence for cardiomyocyte renewal in humans," Science, vol. 324, no. 5923, pp. 98-102, 2009.

[4] S. E. Senyo, M. L. Steinhauser, C. L. Pizzimenti et al., "Mammalian heart renewal by pre-existing cardiomyocytes," Nature, vol. 493, no. 7432, pp. 433-436, 2013.

[5] W. E. Wang, L. Li, X. Xia et al., "Dedifferentiation, proliferation, and redifferentiation of adult mammalian cardiomyocytes after ischemic injury," Circulation, vol. 136, 2017.

[6] L. Tao, Y. Bei, Y. Zhou, J. Xiao, and X. Li, "Non-coding RNAs in cardiac regeneration," Oncotarget, vol. 6, no. 40, pp. 4261342622, 2015.

[7] G. Ruvkun, "Molecular biology: glimpses of a tiny RNA world," Science, vol. 294, no. 5543, pp. 797-799, 2001.

[8] A. Eulalio, M. Mano, M. Dal Ferro et al., "Functional screening identifies miRNAs inducing cardiac regeneration," Nature, vol. 492, no. 7429, pp. 376-381, 2012.

[9] Y. Nakada, D. C. Canseco, S. Thet et al., "Hypoxia induces heart regeneration in adult mice," Nature, vol. 541, no. 7636, pp. 222-227, 2017.

[10] J. D. Fu, S. N. Rushing, D. K. Lieu et al., "Distinct roles of microRNA-1 and -499 in ventricular specification and functional maturation of human embryonic stem cell-derived cardiomyocytes," PLoS One, vol. 6, no. 11, article e27417, 2011.

[11] M. L. Bell, M. Buvoli, and L. A. Leinwand, "Uncoupling of expression of an intronic microRNA and its myosin host gene by exon skipping," Molecular and Cellular Biology, vol. 30, no. 8, pp. 1937-1945, 2010.

[12] X. Li, J. Wang, Z. Jia et al., "MiR-499 regulates cell proliferation and apoptosis during late-stage cardiac differentiation via Sox6 and cyclin D1," PLoS One, vol. 8, no. 9, article e74504, 2013.
[13] B. Dumitriu, M. R. Patrick, J. P. Petschek et al., "Sox6 cellautonomously stimulates erythroid cell survival, proliferation, and terminal maturation and is thereby an important enhancer of definitive erythropoiesis during mouse development," Blood, vol. 108, no. 4, pp. 1198-1207, 2006.

[14] A. L. Clark and F. J. Naya, "MicroRNAs in the myocyte enhancer factor 2 (MEF2)-regulated Gtl2-Dio3 noncoding RNA locus promote cardiomyocyte proliferation by targeting the transcriptional coactivator Cited2," Journal of Biological Chemistry, vol. 290, no. 38, pp. 23162-23172, 2015.

[15] X. Liu, J. Xiao, H. Zhu et al., "miR-222 is necessary for exercise-induced cardiac growth and protects against pathological cardiac remodeling," Cell Metabolism, vol. 21, no. 4, pp. 584-595, 2015.

[16] N. Liu, S. Bezprozvannaya, A. H. Williams et al., "microRNA133a regulates cardiomyocyte proliferation and suppresses smooth muscle gene expression in the heart," Genes \& Development, vol. 22, no. 23, pp. 3242-3254, 2008.

[17] J. L. Morrison, S. Zhang, R. L. Tellam et al., "Regulation of microRNA during cardiomyocyte maturation in sheep," BMC Genomics, vol. 16, p. 541, 2015.

[18] S. Zacchigna and M. Giacca, "Extra- and intracellular factors regulating cardiomyocyte proliferation in postnatal life," Cardiovascular Research, vol. 102, no. 2, pp. 312-320, 2014.

[19] X. Cao, J. Wang, Z. Wang et al., "MicroRNA profiling during rat ventricular maturation: a role for miR-29a in regulating cardiomyocyte cell cycle re-entry," FEBS Letters, vol. 587, no. 10, pp. 1548-1555, 2013.

[20] D. Liang, J. Li, Y. Wu et al., "miRNA-204 drives cardiomyocyte proliferation via targeting Jarid2," International Journal of Cardiology, vol. 201, pp. 38-48, 2015.

[21] J. Chen, Z. P. Huang, H. Y. Seok et al., "mir-17-92 cluster is required for and sufficient to induce cardiomyocyte proliferation in postnatal and adult hearts," Circulation Research, vol. 112, no. 12, pp. 1557-1566, 2013.

[22] J. Shi, Y. Bei, X. Kong et al., "miR-17-3p contributes to exercise-induced cardiac growth and protects against myocardial ischemia-reperfusion injury," Theranostics, vol. 7, no. 3, pp. 664-676, 2017.

[23] Y. Tian, Y. Liu, T. Wang et al., "A microRNA-Hippo pathway that promotes cardiomyocyte proliferation and cardiac regeneration in mice," Science Translational Medicine, vol. 7, no. 279, article 279ra38, 2015.

[24] T. Heallen, M. Zhang, J. Wang et al., "Hippo pathway inhibits Wnt signaling to restrain cardiomyocyte proliferation and heart size," Science, vol. 332, no. 6028, pp. 458-461, 2011.

[25] Y. Yang, H. W. Cheng, Y. Qiu et al., "MicroRNA-34a plays a key role in cardiac repair and regeneration following myocardial infarction," Circulation Research, vol. 117, no. 5, pp. 450-459, 2015.

[26] B. C. Bernardo, X. M. Gao, C. E. Winbanks et al., “Therapeutic inhibition of the miR-34 family attenuates pathological cardiac remodeling and improves heart function," Proceedings of the National Academy of Sciences of the United States of America, vol. 109, no. 43, pp. 17615-17620, 2012.

[27] E. R. Porrello, A. I. Mahmoud, E. Simpson et al., "Regulation of neonatal and adult mammalian heart regeneration by the miR-15 family," Proceedings of the National Academy of Sciences of the United States of America, vol. 110, no. 1, pp. 187-192, 2013. 
[28] E. R. Porrello, B. A. Johnson, A. B. Aurora et al., "miR-15 family regulates postnatal mitotic arrest of cardiomyocytes," Circulation Research, vol. 109, no. 6, pp. 670-679, 2011.

[29] Y. Zhao, J. F. Ransom, A. Li et al., "Dysregulation of cardiogenesis, cardiac conduction, and cell cycle in mice lacking miRNA-1-2," Cell, vol. 129, no. 2, pp. 303-317, 2007.

[30] E. Tzahor and K. D. Poss, "Cardiac regeneration strategies: staying young at heart," Science, vol. 356, no. 6342, pp. 1035-1039, 2017.

[31] M. Ponnusamy, P. F. Li, and K. Wang, "Understanding cardiomyocyte proliferation: an insight into cell cycle activity," Cellular and Molecular Life Sciences, vol. 74, no. 6, pp. 1019-1034, 2017.

[32] K. B. Pasumarthi and L. J. Field, "Cardiomyocyte cell cycle regulation," Circulation Research, vol. 90, no. 10, pp. 1044-1054, 2002.

[33] T. Scholzen and J. Gerdes, "The Ki-67 protein: from the known and the unknown," Journal of Cellular Physiology, vol. 182, no. 3, pp. 311-322, 2000.

[34] F. Beigi, J. Schmeckpeper, P. Pow-Anpongkul et al., "C3orf58, a novel paracrine protein, stimulates cardiomyocyte cell-cycle progression through the PI3K-AKT-CDK7 pathway," Circulation Research, vol. 113, no. 4, pp. 372-380, 2013.

[35] X. Sun, Q. Han, H. Luo et al., "Profiling analysis of long noncoding RNAs in early postnatal mouse hearts," Scientific Reports, vol. 7, article 43485, 2017.

[36] K. See, W. L. W. Tan, E. H. Lim et al., "Single cardiomyocyte nuclear transcriptomes reveal a lincRNA-regulated dedifferentiation and cell cycle stress-response in vivo," Nature Communications, vol. 8, no. 1, p. 225, 2017. 

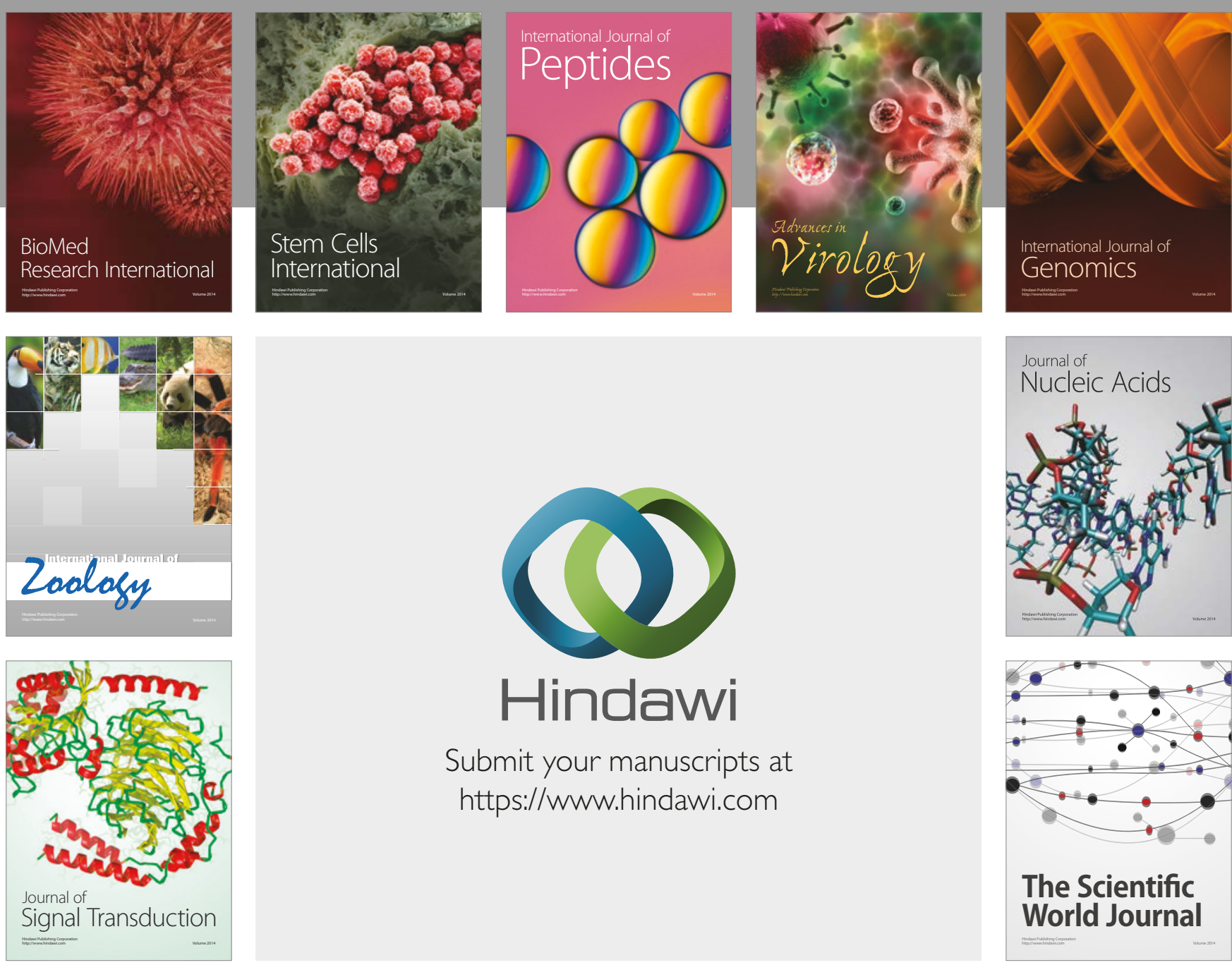

Submit your manuscripts at

https://www.hindawi.com
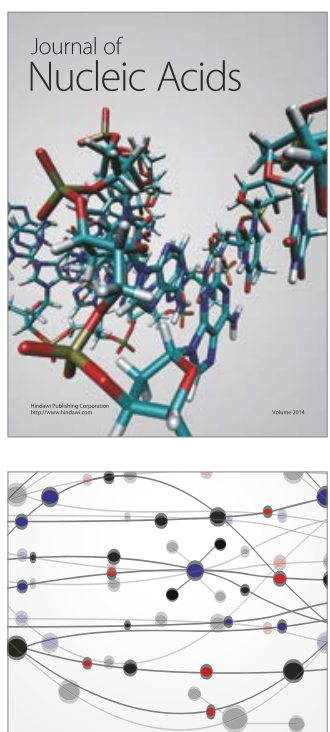

The Scientific World Journal

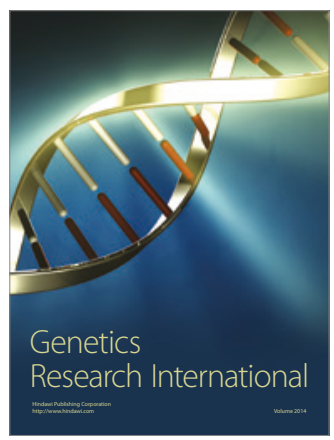

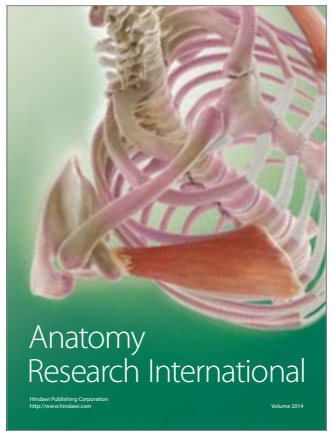

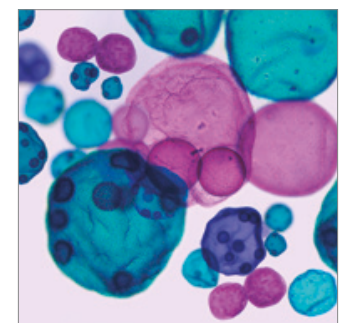

International Journal of Microbiology
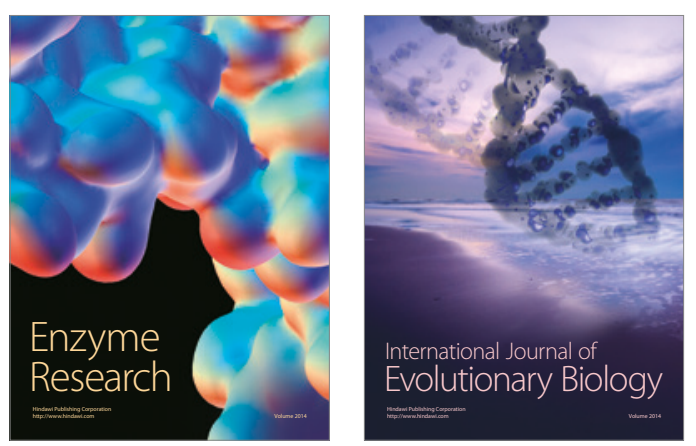
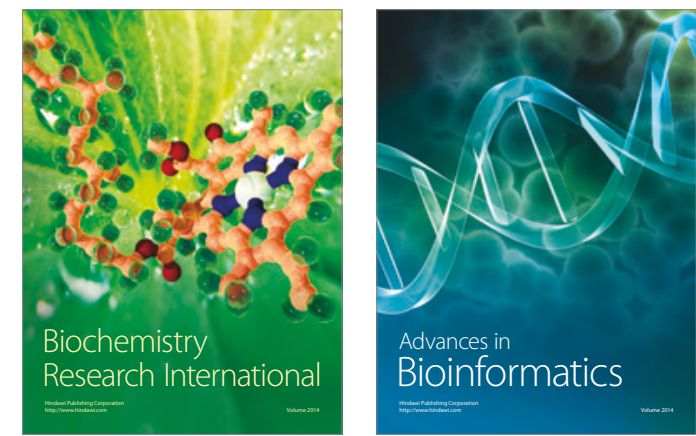

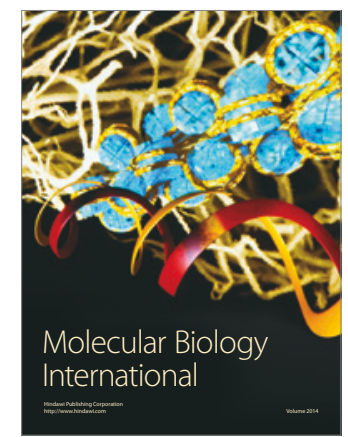

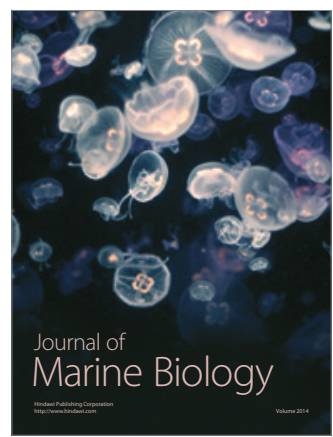

